\title{
Survey: Development and Analysis of a Games-Based Crisis Scenario Generation System
}

\author{
Pisit Praiwattana, Abdennour El Rhalibi \\ Department of Computer Science \\ Liverpool John Moores University, \\ Byrom Street, L3 3AF, Liverpool UK, \\ P.Praiwattana @2014.ljmu.ac.uk, a.elrhalibi@ljmu.ac.uk
}

\begin{abstract}
.
Crisis is an infrequent and unpredictable event which is challenging to prepare and resolve. Serious-game approach proved to provide potential support in training and simulating event of real-world crisis situation to different stakeholders. Yet in practice, the approach meets with difficulty on how to setup and utilize different core components such as asset management, crisis scenario generation, agent simulation, real-world constraints, and the evaluation process to yield beneficial information upon running the system. To address this issue, the key question is what can be done to propose a general crisis game-based framework providing necessary core components while generating evaluation result yielding potential analytical data for a crisis management process. Therefore, in this paper, we aim to review and consolidate the existing research on scenario generation techniques and related crisis simulation framework, then to propose novel solution to combine both processes and to derive a desirable scenario content which is also being validated in the simulation framework based on the JADE multi-agent architecture.
\end{abstract}

Keywords: Crisis simulation framework, Scenario generation, MAS

\section{Introduction}

Crisis can be defined as any event that is, or is expected to lead to, an unstable and dangerous situation affecting an individual, group, community, or the whole society. They can be caused by man-made actions or natural phenomenon or occasional accidents that are difficult to predict (i.e. earthquake, flooding, terrorism, hazardous outbreak, etc.). Crisis management organizations have to handle available resources and facilities to deal with the crisis disaster. These resources change over time in different situations, which causes challenges in preparing the right countermeasure plan, leading to unintentionally experiment via trial and error. Since crises are infrequent, Walker et al. [1] suggests that training from game with relevant real-world environment and well-defined scenario can be beneficial to crisis management personals to understand and prepare for any upcoming emergency situation due to its cost effectiveness in development compared to the setup-cost of real training practice.

Scenario may describe a hypothetical situation that provide a necessary environment setup, initial background of event and final goal to represent a sense of realworld situation in the practical training or test. As regard to of crisis scenario, the possible content structure can be separated into context and crisis. Context represents a pre-incident of crisis situation to enable the understanding of specific environment surrounding the area of event while crisis describes a set of specific events which may lead to dangerous, troubled and challenging situations, with potentially cascading 
effects. To test possible response, the script usually includes unpredictable or probabilistic chain of events that are out of control of any player.

Regarding the gaming technology, the advancement of widely accessible game engine systems such as Unity3D, Ogre3D, Unreal Engine and other related game development tools have improved the pipeline of game development where they reduce the barrier to entry for small team of developers to make games. Subsequently, increase in accessibility to game development application has encouraged many government departments and scientific organizations to apply game as a tool for nonentertainment purposes aiming to provide a mean to give learning experience using gameplay mechanisms such as visualization, interaction, competition, reward system, immersiveness, and so on. Zyda [2] introduced a classification of terms for edutainment game as serious game. By considering the field of crisis management and disaster planning, serious game offers great potential to address the visualization, interaction, communication and assessment of good practice via continuously improving user interface and environment simulation because of the fact that crisis is infrequent and infeasible to be fully demonstrated and experienced within the real world.

Although it is very common that modern serious games stay focus on generating an engaging experience of real crisis situation, developing of the crisis simulation framework for training or decision support system does require a plausible scenario which is often directed from domain experts. This process is time-consuming and often lacks of benchmarks to evaluate the quality of each different unique scenario. Bad design scenario will often lead to unpractical solutions with waste of computing time and training resources. There are several systems which aim to procedurally generate the scenario for training and simulation purpose with a variety of techniques. However, the problem is that the scenario generation system usually is more specific to a dedicated single simulation engine and, thus, is difficult to redeploy on a different system.

The another purpose of representing a crisis situation into a scenario is to be deployed into a simulation system to help answer a question of what is the best possible emergency response regarding to available resource parameters and procedures. A possible solution to the crisis incident is sometimes being represented as a problem of resource allocation and the deployment of crisis manpower. To focus on the process of defining and evaluating a scenario, development of a framework that facilitates the generation of different possible crisis scenario script while simulating its emergency response based on available resources will provide more elaborate understanding of crisis situation and also give a preliminary assessment of crisis scenario and its corresponding plan. The result will be beneficial to the decision support system and to transferring into practical crisis training.

In this paper, we propose a review of existing scenario generation techniques and discuss the context of related crisis simulation systems highlighting the important features of crisis simulation framework. Finally, we propose the development of an interactive game-based crisis scenario simulation framework developed on top of open multi-agent toolkits such as JADE. The reminder of the paper is organized as follows: In section 2, we introduce some existing automated scenario generation systems; in section 3, we review on emergency training and simulation systems; in section 4, we discuss the important features of crisis simulation framework; in section 5, 
we proposed our Crisis Scenario Generation System Framework Design; and, in section 6 , we conclude the paper.

\section{Automated Scenario Generation Systems}

Modern serious games do require a plausible scenario which is often directed from domain experts and it is a very time-consuming process. Automated scenario generation provides major benefits to the manual process by: 1) Content generation can quickly produce on demand scenario with setup and constraints; 2) Computergenerated scenario can be used to supplement human-based scenario quality. For Crisis Management domain, we initially focus on producing a sequence of crisis events while mainly considering: (1) resource management perspective; (2) stakeholder collaboration in solving an emergency situation.

Hullett and Mateas [3] apply a planning technique to generate a firefighter rescue training scenario in the collapsing building area. The system use HTNPlanner with building structure data as input while set a goal to create a situation that satisfy crisis final description, domain knowledge must be defined for planner to allow physical consistency and achieving better plausible result. As a result, the system generates a scenario by filling in content as a sequence of event or activities that are expected to occur and which usually manipulate world environment leading from initial state to the desired goal situation. The trainee is given a role and a set of specific skills to perform in the scenario. The variety of generated scenario is delivered in scale of small, medium and large world setting and it is argued to be better than random probabilistic distribution of element in case of fire situation, damage propagation, and comparable existing systems due to the provision of a domain knowledge consistency model. The main limitation of this system is that the work is tending to encounter a memory shortage during simulating the variation of levels in Medium and Large scale.

Grois et al. [4] developed a SceneGen algorithm for Navy DCTrain System using Noisy Bayesian Network (NOBNs) to search for key-event to satisfying scenario objective using data from knowledge-based in a form of belief network with a penalized likelihood and rejection test to discard non-plausible results. The process is aimed to provide offline-scenario generation. The authors also mention Case-based stochastic perturbation (CBSP) is used to acquire a seed scenario from experts then apply random distribution to manipulate more variation to the original which is likely to bring unreasonable or in-plausible result due to random nature without any testing for plausibility. ScenGen's strength lies in its ability to guarantee the "quality" of each and every scenario it generates through a carefully designed selection bias and it claims to be better in overall performance than Manual design by human subject matter experts (MDHE), Naïve random generation (NRG), and Case-based stochastic perturbation (CBSP) which produce lower-quality scenarios. It defines plausible quality by checking the occurrence of key events according to the desired learning objective. Some learning objective or key event can be occurred and added simultaneously at the same time step. The major drawback of this approach is to require a set of good base human generated scenario as a seed then manipulates them to obtain more variation in the automated results, and in addition the offline generation may lack efficien- 
cy in dynamic crisis simulation system when the setting is reflecting the complexity situation from real-world problem.

Martin et al. [5] proposed an automated scenario generation system which aims to be generic and applicable to any domain specific simulation system. The authors introduced a conceptual mapping approach which is based on 1) training objectives; 2) baseline scenario as a pre-defined ideal parameter scenario; and 3) scenario vignettes that is a complexity modification of scenario such as weather, light, and etc. The main objective and additional data such as weather condition (vignette) setting are composed in an XML file and generate the scenario variation using scripted functional Lsystem which are similar to shape grammar in procedural modelling but define the syntax rule to represent scenario elements. This system allows generic automated domain-independent scenario generation with different simulation framework. However, the disadvantage is that it requires extra work on developing a rule for FLSystem but claimed that it is one-time requirement for new training domain and it is reusable in similar domain setting. The difficulty on generating a rule is also arguable.

Zook et al. [6] introduce a combinatorial optimization approach to scenario generation to deliver the requisite diversity and quality of scenarios while tailoring the scenarios to a particular learner's needs and abilities for military training in virtual environment which is opposite to the planning approach. The main scenario generation based is on a genetic algorithm to search for a best solution; reading in authorspecified domain knowledge, the details and the type of possible events and requirement in scenario, and constraint order on events. The process works by considering instant event template for the scenario at random location; Mutating the parameter of random chosen scenario; Applying cross-over operation, to create new sequence of events to improve the quality of scenario for the next iteration. The authors proposed evaluation in an interesting and effective way: 1) quality of solution at run-time; 2.) the diversity of scenario as function of running time, 3) performance of trainee and appropriateness of difficulty level when training on generated scenario. The result from their evaluation is to generate a unique scenario compared to planner generation approach, this technique based on a combinatorial optimization provides lowerquality solution initially, but explores multiple different regions in the solution space containing high-quality solutions and so refines multiple distinct scenario that meet provided learning objectives rather than explore variation on same high-quality scenario. In practice, planner is expected to yield a high-quality tailored scenario early and produce several scenarios of roughly equivalent quality that are very similar on a several high-quality scenario. The authors states the requirement of virtual technology for scenario training is vital to incorporates learner attributes and theoretically lead to more effective training as learner have greater opportunities to train more on relevant scenario. The major drawback is that the generation requires a predefined small element of events to be tailored into scenario and initial input of learner model for suitable evaluation fitness function for the genetic algorithm.

Different computational approaches have been proposed for scenario generation system. From the reviews, the combinatorial optimization search and planning techniques are efficient to produce a variety of quality scenario. The former is generic optimization approach working by evaluating a set of function to determine a necessary event elements of given scenario. It works best for a training aspect since these parameters such as scenario length, constrained sequence structure and a learner mod- 
el are available to achieve a relevant and distinct result each time; the latter is deploying a planner to fill in between different key events based on the event precondition and constraints. The planner approach is simpler to design and control since the knowledge representation is concise using a formal planning domain definition language such as PDDL. The lack of variation by using planner is still arguable since there are several example system in digital story telling generation successfully applying such a method to order a story priori based on given setting element of dramatic arc [7]. While the seed approaches which blended a human-created element and automated searching process seem to be more effective in generating high-quality result, the lack of variation and time-consuming aspect make this technique less preferable in representing a general crisis simulation framework. For a heuristic approach, the creating of scenario is formed by evaluating a subcomponent of scenario to the given heuristic function. The given subcomponent will be kept in a final scenario if it passed the threshold although this method seems to deliver an effective scenario, it is computing inefficient. For our framework, we will employ the planning approach due to its simplicity in design for knowledge representation of crisis scenario rather than developing a learner model.

In this section, we have reviewed some of the proposed techniques on scenario generation system. We will continue discussing the crisis training and simulation system in the section 3 .

\section{Crisis simulation systems}

Following the review of the work on crisis generation, we propose in this section to review the related works on crisis simulation system.

The appropriateness of computer simulation for crisis emergency response can be observed by its category of usage: 1) to provide experience for training and entertainment using: a) virtual equipment; b) gaming simulation; and c) combining a real system and simulation (to for e.g. enhance operational skill); 2) to perform experiments including education, understanding and decision support system (DSS) [8].

Agent-based simulation (ABS) has been used consistently with emergency response simulation due to the flexibility to model different situations with real-world complexity knowledge. An Agent is often considered as representation of human individual capable with a set of available skills involved in the domain, typically either civilian or rescuer, but sometimes it also represent non-human entity such as vehicles and building. An Agent is usually proactive and will be used to achieve some goal, and it is reactive in response of change in the environment [9]. An Agent is often scripted with different set of behavior depending on the given role and defined rules of system. The system is often designed to emulate how the events in the realworld unfold and then determine the appropriate response plan for the agents. ABS is best suited for modelling attack on transport, attack on crowded place, pandemic human disease and natural disaster such as flooding [10]. Several existing systems work around the concept of ABS for large scale emergency response and some aim to develop a serious game for crisis simulation system with modern gaming technology for practical training and also study the effective response of crisis plan.

Metello et al. [11] designed an emergency simulation game based on serious game technique aiming to represent observable crisis situations rather than just a representa- 
tion of crisis in the learning game. This system helps to demonstrate an emergency plan in finding a flawed procedure during any given scenario such as oil leakage by allowing the trainee to interact with a simulation system which events has been fired to the world environment over time and providing a feedback by rendering the effect on the screen.

Schoenharl et al. [12] demonstrated simulation of a multi-agent system WIPER (wireless integrated phone-based emergency response) corresponding to real GIS visualizing geographic terrain with each mobile phone users having a movement activities tracked by tower cellular segments in the area for a real-time response. A realtime data source provides real-time data regarding cell-phone usage from cell-phone providers. Using a historical data source (a repository of normal cell-phone usage), a detection and alert system detect possible anomalies in cell-phone usage patterns. The system also operates by taking a batch of different agent movement activities for simulation aiming to mimic the crisis event which are: 1.) a flee event where every agent move away in disturbance; 2) a flock event where agent is trying to move as a mob (grouping); 3) a jam event where each agent is moving toward their specific goal but is constrained as in traffic jam. Their system can simulate a density of agents as population and measure traffic activity which represents the actual cell segment for a better understanding of the crisis situation.

Saoud et al. [13] describe a multi-agent based approach for modeling a simulation (SimGenis) to design optimal, efficient, and appropriate rescue strategies, based on the initial state of victims, number of rescuers, and method of communication between rescuers (electronic or paper). More precisely, the aim of the research was to determine how the response to a dynamic large-scale emergency depends on the use of a centralized and decentralized collaborative rescue strategy with applying heuristic algorithm on each agent and component of the simulator while testing using seven configurations with 300 agents for victims and rescuers. The optimal response for the simulation is derived from the total number of victims, initial state of the victims and the total number of rescuer (doctor, firefighter, and nurse). The results from the study states that there is no best unique rescue scenario and it is hard to predict depending on the disaster characteristic.

Takeuchi [14] introduced a Robocup Rescue which is using the 1995 Kobe earthquake as the original test scenario. The system is aiming to represent the disaster situation sensory information then to incorporate the agent-simulation system to mitigate disaster and encourage large scale research collaboration by holding an annual competition since 2001. The response is based on optimization of the design and implementation of better action selection method to maximize the objective function regarding the number of individual, proportion of remaining health to initial health point, and proportion of unburnt area of building. In addition, with the nature of multi-objective in crisis response, a vector-based score has been proposed to compare different responses strategies [15].

The AROUND project: Adapting robotic disaster response to developing countries [16] introduced the use of robots for observation task by the information gathered by robot is fed to spatial decision support system (SDSS), which uses agent-based model to predict the outcomes of possible course of action by human rescue teams. In AROUND, optimal agent behavior mimics that of the real-world rescuers they represent. The test scenario being used is modeled after an earthquake in the city of Hanoi 
(Vietnam) from 1935 and 1983. The project has some similarities to Robocup Rescue in the deploying a usage of robot in order to reducing a disaster damage in developing country. In AROUND, behavior is also modeled using a parameterized utility function, which is used for action selection and has similarity to real-life behaviors.

PLAN-C (planning with large agent-networks against catastrophes) is also another ABS developed to predict the behavior of individual and collectively in large-scale emergency such as terrorist attack [17] [18]. The simulation scenario is modeled after a possible terrorist attack which simulates the first 50 hours following a Sarin chemical attack on Manhattan Island. The emergency response is represented as finding a solution to a multi-objective optimization problem (MOOP) since there is no best single optimum solution in the nature of disaster. The Pareto-optimal trade-off between different parameters is being discovered. The parameters being observed can be varied such as hospital resource level (consumable such as drug and medicine; recoverable such as bed and personals). The result is that increasing the number of resources will rapidly reduce the fatalities; however, up on passing some thresholds, the fatalities will not lessen even with more resources available. Still, the system provided an analysis result of civilian behavior on attending the nearby hospital regarding the effectiveness of hospital to admit and release the appropriate number of patient to reduce the most fatalities.

EpiSimS [19] [20] is also another ABS developed to study the optimized parameter of resource and procedures in a simulated SmallPox attack model and influenza outbreak. The analysis result from the simulation is that the rate of death is directly influenced by how quickly the infected patients can isolate themselves from the rest of population (society). Whereas, the study of deploying an official response such as mass evacuation, vaccination and quarantine to control the spread of smallpox is less effective on the rate of death.

From reviews, the proposed crisis simulation systems are aiming to train and study the effective result of deployed procedures during an emergency situation. The agentbased model is a core component to represent a complex and realistic system in the occurring crisis by modeling an agent with corresponding roles such as victim, crisis personal and facilities. The multi-objective optimization search is a common approach to investigate a best trade-off response to reduce the fatality or damage. The test scenario usually model after the large scale crisis occurred in the local region of the project. In this section, we have reviewed some of the proposed system on emergency simulation systems. We will continue discussing the overall of crisis framework in the section 4.

\section{Discussion on Crisis Simulation Framework}

So far in the available literature, there are several similarities in the development of a crisis simulation framework regarding the preparation stage. The majority of the simulation system aim at representing the real-word knowledge then reproduces the crisis scenario based on historical records for devising a better decision support system and plan; and also to train the crisis personal for any upcoming event for a proper response.

During Real-time crisis situation, simulation framework such as WIPER applies the mobile network GIS information system to provide a realistic and real-time re- 
sponse for decision support simulation. Also for the preparation of study of crisis events by varying the different resource level, system such as PLAN-C can provide an analytical study of improved response on investigating some component of behavior. In addition, a new behavior which is not constrained to be human resemblance can be investigated as to maximize the evaluation function in Robocup Rescue. The similarities is that each simulation system is deployed using the agent-based model framework to simulate the behavior of individual such as victim and crisis personal (e.g. firefighter, doctor) then measure the parameter of objective function such as fatality rate as utility. The algorithm is often based on optimization of the objective function to derive a best trade-off practice of parameters on resource and procedures. The representation of world environment is often in a form of grid of cells such as in SimGenis and PLAN-C but later on the requirement to achieve realistic simulation it was necessary to incorporate GIS information input.

The simulation system can also benefit from deploying a scenario generation procedure. Each type of real-world complex situation requires a large and, sometimes, inaccessible information. In order to prepare and study a generic crisis and disaster situation from small-to-large scale, the procedural generation of scenario is desirable. In training, the procedural generation of scenario is still being an active study area. There are several common techniques on the process: Seed approach which blends the human generated sub-scenario with an automated system to append and manipulate the sequence of events for more variability such as SimGen, and is also a distinct method to derive a quality scenario. Planning, by using planner to fill in the inbetween event from each key constrained events, is also a concrete method for generating consistent scenario since the domain knowledge is being scripted into the available condition of planner actions which also share similar usage in digital story telling system [7]; Functional-L system, with a requirement of developing a subsequent set of rule for conducting a scenario, the difficulty may rely on how to identifying rules that would produce a suitable set of scenario with varying result.; and a generic optimization approach, algorithms that apply mutation and cross-over to the human generated sub-key events to obtaining a unique scenario result based on the specific given parameter and learner model as fitness evaluation function.

Although, there is no distinct best solution on how to generate crisis scenarios, there are desirable components that the automated generation system should provide: 1.) To allow scenario generation to operate with different simulation platform using a general data representation such as XML; 2.) To incorporate an external simulation model knowledge such as fire, flood, epidemic and weather (e.g. tornado and earthquake); 3.) To generate the scenario based on GIS vector information which would be sufficient to represent the environment of complex real-world problem; and 4.) To allow interaction with user during a generation process and provide accurate desirable result during generation and evaluation of scenario.

While there are different techniques in generation of scenario and simulating of crisis situation, there is not much novel solution to combine both processes and to derive a desirable scenario content which is also being validated in the simulation framework. In next section, we propose a scenario generation framework based on the JADE multi-agent architecture which is aiming to incorporate the scenario generation technique and crisis simulation agent-based model into a unify system. 


\section{Crisis Scenario Generation System Framework Design}

The crisis scenario generation framework is composed of many components. Figure 1 represents a high level architecture of our crisis scenario generation framework. In the following section, we will introduce these components respectively.

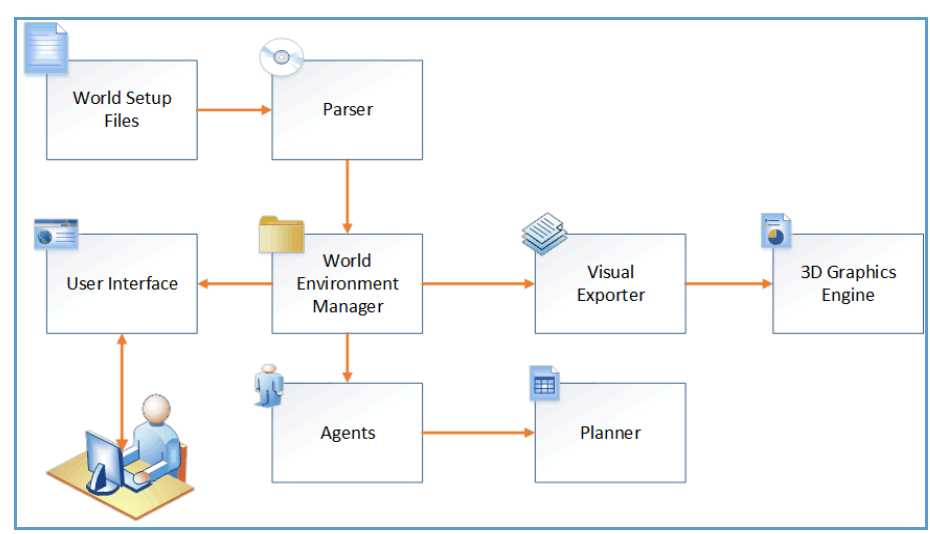

Fig. 1. Crisis scenario generation framework architecture

\subsection{Parser Component}

Setup information including different scope of scenario world location, available resources, possible set of actions, constraints, possible set of events' description, and related configuration keys. The purpose of this component is to parse the necessary files to initializing the base representation of the system world environment. Moreover, it is possible to create an API for importing a world-setup from different framework. This feature will be revisited in the later stage of development.

\subsection{World Environment Manager Component}

The world environment manager has the main task to handle the representation of centralized scenario world scene. It is responsible to maintaining the knowledge of the world stage based on any change from agents' actions or event triggering. It also allocates the information regarding the current situation in a scenario to agent manager with planner instance. Moreover, the world environments will coordinate and respond to agent's request for executing an action in the environment. Any conflict from agents' request will be resolved via this module rather than direct negotiation between each agent. In implementation detail, World Environment Manager may be operating as central agent which stores the world environment data structure and allow the execution of an action to manipulate the existing object variables in environment.

The sub-components of world environment manager are described as in the following:

- World Scene: The world scene component will hold the list of scenario knowledge and available resources in the current environment state of the world regarding the setup location and being updated by incrementing time steps. It is a central data structure which allows allocating of world stage knowledge into any requested component. 
- Event Manager: The event manager component utilizes a pool of events with probabilistic model. It fires a sequence of events to conduct the scenario incident, which can be deterministic to representing a cascading effect of specific real-world situation depending on the proper setup files. This component will be directly monitoring the world scene and its incident world event will be allocated with corresponding world-scene information to the requested agent.

- Time Manager: This component handles keeping track of the time in the virtual simulation for conveniently synchronizing and calculating the action duration.

- Action Capability: All possible agent actions will be stored in this module with their specific precondition to execute such as requester role, available resource condition, and time duration.

- Statistic and Evaluation Manager: This component will keep statistic result data regarding each crisis incident in the generated scenario according with its executing plan providing a mean to evaluate the utility from outcome of different plan or decision.

- Output generator: It generates the human readable representation of the current world state for being used in report and interacting with user

- History Manager: the history component will keep track of actions requested in each state of the world with meta-data for respective results in the world scene. This component served as an optional module to load/rollback the specific checkpoint of the world state which will contribute into framework novelty later on.

- Conflict Manager: The conflict resolver component will have an authority in resolving the conflict between agents' request. Currently, every action is not generated as a partial-order plan so there is likely to have no conflict. However, the necessity to have a dedicated component will become handy in extending the feature of framework.

\subsection{User Interface}

Current world information sent from the world manager output module will be displayed in this user interface component. User can observe the development of generating scenario step-by-step in a sequence of narrative log text. However, the current design does not focus on visualizing the virtual environment to the user via this channel directly. User may use this component to configure the option of the scenario setup before starting the actual simulation and also assign a preference choice for agent decision making if applicable.

\subsection{Agent Component}

The framework will elaborate the world scene and scenario by applying a multiagent framework solution as each agent represents an actor in the scenario. In general, crisis management situation normally includes different tiers of decision making chain of command and, sometimes, crisis scenario can be represented in according to different scope of scenes whether a city-area event or in-a-building floor layout scene. To address this model, agents may be allocated into separated level of abstract representation which can be defined as crisis manager, crisis facility/station, and crisis personal team. The hierarchy in chain of command will be deployed from manager to personal tier respectively. A simplify assumption will be made to indicate an actual sequence of agents' behavior, which greatly reduces system complexity. The representation of agents' type is described as in the following:

- Decision Making Agent (DMA): This agent acts as a high-level decision making unit, crisis manager. In general, the decision-making agent, crisis manager, will be equipped with an in- 
stance of planner to generate plans according to the given world state and event notified from world environment manager. While using utility function preference, High-level plan will focus on allocation of resource, task assignment, optimization then being sent to control agents.

- Control Agents (CA): Control agent represents a facility unit, crisis facility. Being allocated the resource and task from DMA, this unit may evaluate resources with the given task in the case that the additional resource is required. If applicable, CA instantiate a field agent unit with setup of necessary resources and task.

- Field Agents (FA): This agent represents personal teams with resources. FA will perform direct request to execute an action with a world environment manager component.

Both CA and FA will use planner instance to perform planning with different level of scope from DMA, which focuses on essential actions that allow them to solve the given task. In addition, the agents will not directly negotiate between each other but directly report the result of current active action and its success or failure to the higher-tier agent then wait for further instruction. This allow the decision making agent to clearly monitor the assigned action's outcome. If there is a failure in the assigned action, it can evaluate the current situation and apply replanning algorithm if applicable.

Agents will be developed on top of JADE, multi-agent framework using standard agent communication language, ACL [21]. This technology will provide ease of message transferring and scalability as agents can be host on different machines on a distributed system.

\subsection{Planner}

Planner component allows the generation a plan consisting of sequence actions related to a given world state, allocated resources, and possible actions. This world information must be preprocessed into PDDL format then being stored in the local KBS of the Planner. The external planner instance will be deployed as in the current state to fasten the development of framework. We currently select a planner using forward chaining state space search to reach a goal with heuristic function, FF_Metric [22]. The constraint in resources, agent's actions, and goal's condition will be defined and solved as a constraint satisfaction solution [23].

It is important to note that there is no permanent decision on selecting a planner which can be revised in later stage. In the future work, this component may be designed with new algorithms providing better flexibility in creating a crisis plan with PDDL4J Library. Monitoring algorithm and replanning algorithm are currently designed to handle execution of plan list resulted from a planner in general. Further design will include time duration boundary variable to provide more realistic replanning approach with dynamic and changing real-world situation. Figure 2 depicts the planner architecture. 


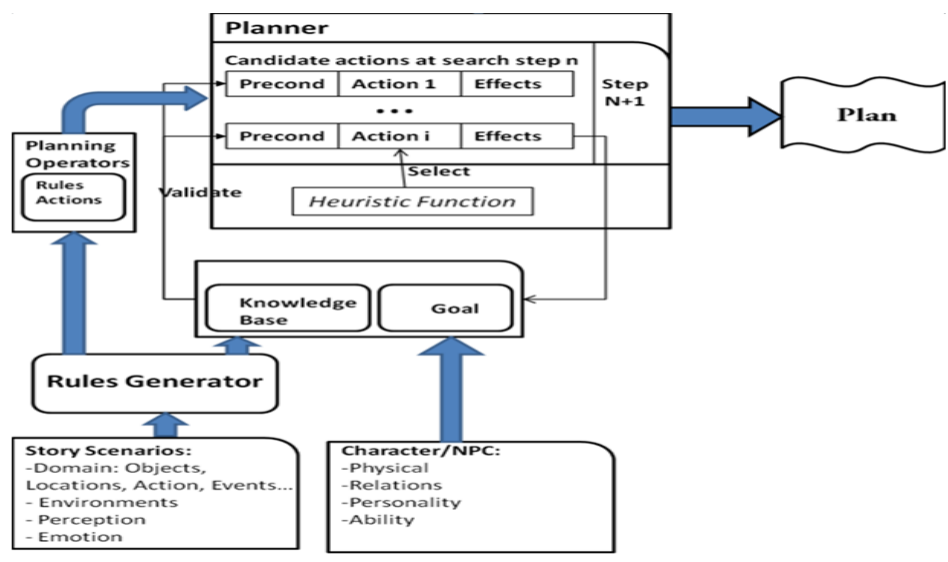

Fig. 2: Planner Architecture

\subsection{Visual Exporter}

This component will be used for the frameworks with external graphics engine. The information of world representation must be formatted into a common data extension such as XML or COLLADA, which can be efficiently parsed for visualizing purpose later on. In the early state of current design, exporting data should contain a data structure representing each simulation step of world scene state. In future extension, the framework may import a world data then generate an incident event scenario with a sequence of solution plan. This information may be exported into game engine to use as a sequence of event simulation rather than one-time step of world scene.

\subsection{D Graphics Engine}

This component aims to demonstrate how the frameworks can connect to external 3D graphics engines such as Unity3D and other similar engines. The crisis scenario simulation will be represented as $2 \mathrm{D}$ or $3 \mathrm{D}$ visual data in which the graphics representation of each element can be mapped from exported data of the world scene. This component is currently an optional extension upon completing the final implementation. Directly establish a socket communication with the 3D engine may be tested for real-time performance evaluation.

\section{Framework scalability evaluation}

Currently, the proposed framework is in an initial development stage, the core components involving world environment, agents, and planning have been implemented. We summarized an evaluation in this section.

To test a performance and scalability, we proposed increasing number of active agents in the system while measured a total duration to solve the given scenario tasks and a plan generation time For Testing scenario, we implemented a basic firefighter domain which describe fire situation where a world object is set on-fire. There are 4 
basic operations which are move-to, pick-up-a-supply, extinguish-a-fire and rest while there are only one type of actor and facility, fireman and firestation

The number of agents are instantiated with a scene of fixed size world objects in a single event. The time reported are run in a Laptop with Core i7-5500U @2.40GHz, 2 Cores with RAM 16GB (10GB Available), and Window 10.

Our result are illustrated in Figure 3 . We test initially with single agent and then 2, $5,10,20,30,50,100$ respective in simultaneous instantiation to solve a world-event problem with 20 on-fire objects.

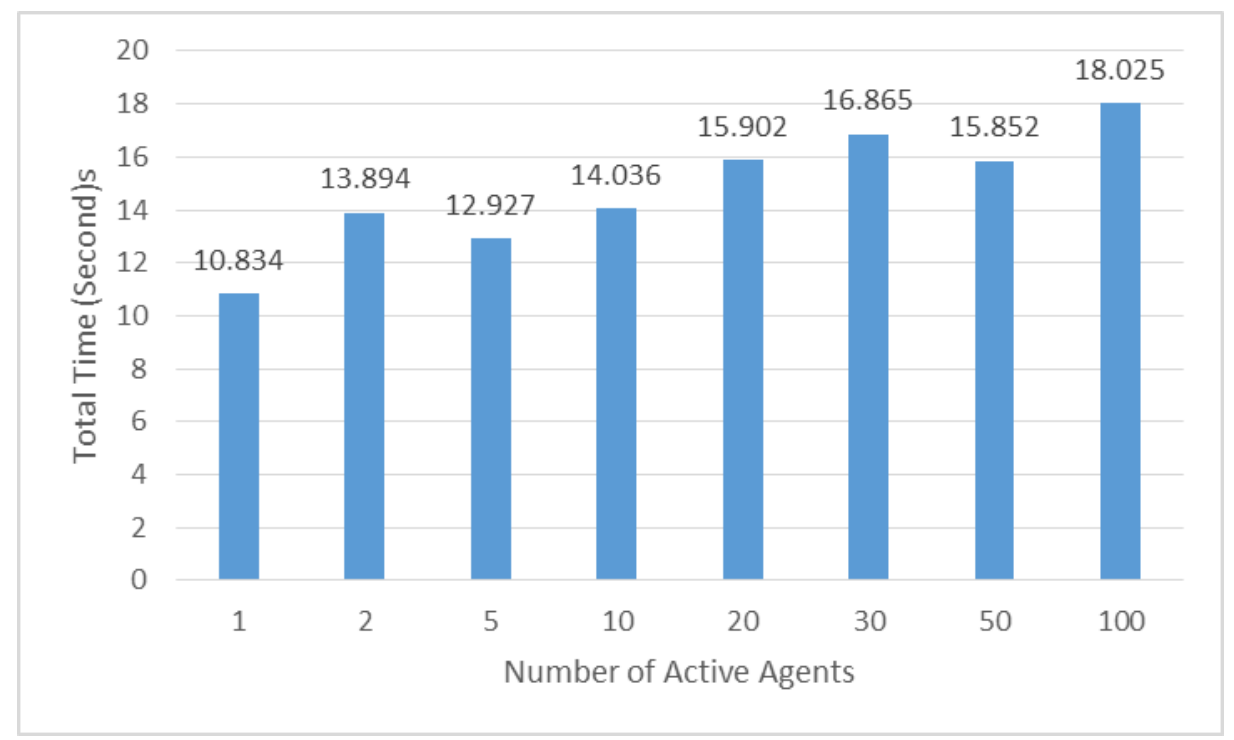

Fig. 3: Computation time of simultaneously solving a world event

The result indicates that increasing the number of agents will require more time due to the fact that they generated more collision in action execution in the environment such as trying to execute an action on the same object. Only one agent will success while another fails triggering replanning process. This situation may lead to studying of opportunistic competition between each agent and invention of more efficient planning and replanning algorithm for multi-agent system.

Currently, the evaluation is based on a single machine rather than hosting an agent on different machines communicated on JADE environment which could improve stability and reduce execution time in larger world setup.

The re-evaluation will perform after the implementation of command-tier agent such as fire station to simulate resource management environment in future version.

\section{Conclusion}

In this paper, we discuss a review on existing work regarding automated scenario generation technique and related crisis simulation framework then present a design of crisis scenario simulation framework developed on top of open multi-agent framework such as JADE to provide a new solution incorporating techniques based on 
planning in both area. This approach has a potential to provide a large variety of study and test environment to validate theory and plan in different situation on across domain especially crisis management. Further, it will allow the evaluation of trade-off between each decision on different perspective as stated in separated tiers of agent. While in regarding the scalability problem for simulation, the multi-agent framework enables a larger scale of computation with connecting more platforms as a container to the environment;

In the future work, we will continue the implementation of base simulation framework and provide more evaluation on result of different scenario setup with different tiers of agent. We will also develop an automated scenario generation on top of the simulation framework to validate and provide diversity of representation of crisis scenario.

\section{References}

1. Walker, W. E., Giddings, J., \& Armstrong, S. (2011). Training and learning for crisis management using a virtual simulation/gaming environment. Cognition, Technology \& Work Cogn Tech Work, 13, 3, 163-173.

2. Zyda, M. (2005). From visual simulation to virtual reality to games. Computer, 38, 9, 25-32.

3. Hullett, K., Mateas, M. (2009). Scenario generation for emergency rescue training games. 4th International Conference on Foundations of Digital Games - FDG '09.

4. Grois, E., Hsu, W. H., Voloshin, M., \& Wilkins, D. C. (1998). Bayesian network models for generation of crisis management training scenarios. In AAAI/IAAI, 1113-1120.

5. Martin, G., Schatz, S., Bowers, C., Hughes, C., Fowlkes, J., Nicholson, D. (2009). Automatic Scenario Generation through Procedural Modeling for Scenario-Based Training. The Human Factors and Ergonomics Society Annual Meeting. 53, 1949-1953.

6. Zook, A., Riedl, M. O., Holden, H. K., Sottilare, R. A., and Brawner, K. W. (2012). Automated scenario generation: Toward tailored and optimized military training in virtual environments. 7 th International Conference on the Foundations of Digital Games.

7. Porteous, J., Cavazza, M., \& Charles, F. (2010). Applying planning to interactive storytelling: Narrative control using state constraints. ACM Transactions on Intelligent Systems and Technology 1, 2, 10.

8. Ören, T., Longo, F., (2008). Emergence, Anticipation and Multisimulation: Bases for conflict simulation. $20^{\text {th }}$ European Modeling \& Simulation Symposium (EMSS), 546-555.

9. Wooldridge, M., Jennings, N. R. (1995). Intelligent agents: Theory and practice. Knowl. Eng. Rev. 10, 2, 115-152.

10. Challenger, R., Clegg, C. W., Robinson, M. A. (2009). Understanding crowd behaviours: Simulation tools. U.K. Cabinet Office.

11. Metello, M. G., Casanova, M. A., \& de Carvalho, M. T. M. (2008). Using Serious Game Techniques to Simulate Emergency Situations. In GeoInfo, 121-182.

12. Schoenharl, T. \& Madey, G. (2011). Design and implementation of agent-based simulation for emergency response and crisis management. Journal of Algorithms \& Computational Technology 5, 4.

13. Saoud, N. B. B., Mena, T. B., Dugdale, J., Pavard, B., \& Ahmed, M. B. (2006). Assessing large scale emergency rescue plans: an agent based approach. Int J Intell Control Syst, 11(4), 260-271.

14. Takeuchi, I. (2005). A massively multi-agent simulation system for disaster mitigation. First International Workshop on Massively Multi-Agent Systems I (MMAS '04), Springer, Berlin, 269.

15. Siddhartha, H., Sarika, R., Karlapalem, K. (2009). Score vector: A new evaluation scheme for RoboCup Rescue simulation competition.

16. Boucher A., Canal, R., Chu, T., Drogoul, A., Gaudou, B., Le, V., Moraru, V., Van Nguyen, N., Vu, Q., Taillandier, P. (2009). The AROUND project: Adapting robotic disaster response to developing countries. IIEEE Workshop on Safety, Security \& Rescue Robotics, Washington, D.C., 1-6.

17. Mysore, V., Narzisi, G., Mishra, B. (2006). Agent modeling of a Sarin attack in Manhattan. First International Workshop on Agent Technology for Disaster Management, 108-115.

18. Narzisi, G., Mysore, V., \& Mishra, B. (2006). Multi-objective evolutionary optimization of agentbased models: An application to emergency response planning. IComputational Intelligence, 228-232. 
19. Barrett, C. L., Eubank, S. G., \& Smith, J. P. (2005). If smallpox strikes Portland, Scientific American Journal, 292, 3, 54-61.

20. Mniszewski, S. M., Del Valle, S. Y., Stroud, P. D., Riese, J. M., \& Sydoriak, S. J. (2008, April). EpiSimS simulation of a multi-component strategy for pandemic influenza. In Proceedings of the 2008 Spring simulation multiconference, 556-563.

21. FIPA Agent Communication Language (ACL) specification. Retrieved from http://www.fipa.org/repository/aclspecs.html [Accessed 31 January 16].

22. Hoffmann, J. (2003). The Metric-FF Planning System: Translating “Ignoring Delete Lists" to Numeric State Variables. Journal of Artificial Intelligence Research, 291-341.

23. Yokoo, M. (2001). Constraint Satisfaction Problem. In Distributed Constraint Satisfaction, Springer Berlin Heidelberg, 1-45. 